\title{
Erratum to: Stabilization of a Supersaturated Solution of Mefenamic Acid from a Solid Dispersion with EUDRAGIT ${ }^{\circledR}$ EPO
}

Taro Kojima $\cdot$ Kenjirou Higashi $•$ Toyofumi Suzuki $•$ Kazuo Tomono $•$ Kunikazu Moribe $•$ Keiji Yamamoto

Published online: 3 February 2012

(C) Springer Science+Business Media, LLC 2012

\section{Erratum to: Pharm Res}

\section{DOI 10.1007/s11095-011-0655-7}

The following note was omitted from the article: Authors Taro Kojima and Kenjirou Higashi contributed equally to this work. In addition, the corresponding author should have been listed as Kunikazu Moribe, email: moribe@p.chiba-u.ac.jp.

The online version of the original article can be found at http://dx.doi.org/ | 0. | 007/s | |095-0 | |-0655-7.

T. Kojima $\cdot$ K. Higashi $\cdot$ K. Moribe $(\varangle) \cdot$ K. Yamamoto

Graduate School of Pharmaceutical Sciences, Chiba University

I-8-I, Inohana, Chuo-ku, Chiba-shi

Chiba 260-8675, Japan

e-mail: moribe@p.chiba-u.ac.jp

K. Yamamoto

e-mail: yamamotk@p.chiba-u.ac.jp

T. Suzuki $\cdot$ K. Tomono

Department of Pharmaceutics, School of Pharmacy, Nihon University

7-7-I Narashinodai, Funabashi-shi

Chiba 274-8555, Japan 